\title{
Integrated intravascular ultrasound and optical coherence tomography technology: a promising tool to identify vulnerable plaques [INVITED PAPER]
}

\author{
Jiawen Li ${ }^{\#}$, and Zhongping Chen* \\ Beckman Laser Institute, University of California, Irvine, 1002 Health Sciences Road East, Irvine, CA 92612, USA \\ \# The author is currently working at the Optical+Biomedical Engineering Laboratory, the University of Western \\ Australia. \\ ${ }^{*}$ Correspondence and requests for materials should be addressed to Z.C. ( e-mail: z2chen@uci.edu )
}

\begin{abstract}
Heart attack is mainly caused by the rupture of a vulnerable plaque. IVUS-OCT is a novel medical imaging modality that provides opportunities for accurate assessment of vulnerable plaques in vivo in patients. IVUS provides deep penetration to image the whole necrotic core while OCT enables accurate measurement of the fibrous cap of a plaque owing to its high resolution. In this paper, the authors describe the fundamentals, the technical designs and the applications of IVUS-OCT technology. Results from cadaver specimens are summarized, which indicated the complementary nature of OCT and IVUS for assessment of vulnerable plaques, plaque composition, and stent-tissue interactions. Furthermore, previously reported in vivo animal experiments are reviewed to assess the clinical adaptability of IVUS-OCT. Future directions for this technology are also discussed in this review. (C) 2016 Samara State Aerospace University (SSAU).
\end{abstract}

Keywords: Optical coherence tomography, ultrasound, multi-modality imaging, intravascular imaging, vulnerable plaques, cardiology, intravascular OCT, IVUS, integraged IVUS/OCT.

Paper \#2865 received 2015.11.29; revised manuscript received 2015.12.25; accepted for publication 2015.12.27; published online 2016.02.01.

\section{References}

1. K. Okrainec, D. K. Banerjee, and M. J. Eisenberg, "Coronary artery disease in the developing world," American Heart Journal 148(1), 7-15 (2004).

2. J. G. Kips, P. Segers, and L. M. Van Bortel, "Identifying the vulnerable plaque: A review of invasive and noninvasive imaging modalities," Artery Research 2(1), 21-34 (2008).

3. J. Sanz, and Z. A. Fayad, "Imaging of atherosclerotic cardiovascular disease," Nature 451(7181), 953-957 (2008).

4. D. Vancraeynest, A. Pasquet, V. Roelants, B. L. Gerber, and J.-L. J. Vanoverschelde, "Imaging the Vulnerable Plaque," Journal of the American College of Cardiology 57(20), 1961-1979 (2011).

5. J. R. Davies, J. H. F. Rudd, P. L. Weissberg, and J. Narula, "Radionuclide Imaging for the Detection of Inflammation in Vulnerable Plaques," Journal of the American College of Cardiology 47(8 SUPPL.), C57-C68 (2006).

6. T. Kubo, T. Imanishi, S. Takarada, A. Kuroi, S. Ueno, T. Yamano, Y. Matsuo, T. Masho, H. Kitabata, K. Tsuda, Y. Tomobuchi, and T. Akasaka, "Assessment of Culprit Lesion Morphology in Acute Myocardial Infarction," Journal of the American College of Cardiology 50(10), 934-939 (2007).

7. T. Sawada, J. Shite, H. M. Garcia-Garcia, T. Shinke, S. Watanabe, H. Otake, D. Matsumoto, Y. Tanino, D. Ogasawara, H. Kawamori, H. Kato, N. Miyoshi, M. Yokoyama, P. W. Serruys, and K.-I. Hirata, "Feasibility of combined use of intravascular ultrasound radiofrequency data analysis and optical coherence tomography for detecting thin-cap fibroatheroma," European Heart Journal 29(9), 1136-1146 (2008). 
8. K. Fujii, H. Hao, M. Shibuya, T. Imanaka, M. Fukunaga, K. Miki, H. Tamaru, H. Sawada, Y. Naito, M. Ohyanagi, S. Hirota, T. Masuyama, "Accuracy of OCT, grayscale IVUS, and their combination for the diagnosis of coronary TCFA: An ex vivo validation study," JACC: Cardiovascular Imaging 8(4), 451-460 (2015).

9. M. Kawasaki, B. E. Bouma, J. Bressner, S. L. Houser, S. K. Nadkarni, B. D. MacNeill, I.-K. Jang, H. Fujiwara, and G. J. Tearney, "Diagnostic Accuracy of Optical Coherence Tomography and Integrated Backscatter Intravascular Ultrasound Images for Tissue Characterization of Human Coronary Plaques," Journal of the American College of Cardiology 48(1), 81-88 (2006).

10. J. Rieber, O. Meissner, G. Babaryka, S. Reim, M. Oswald, A. Koenig, T. M. Schiele, M. Shapiro, K. Theisen, M. F. Reiser, V. Klauss, and U.Hoffmann, "Diagnostic accuracy of optical coherence tomography and intravascular ultrasound for the detection and characterization of atherosclerotic plaque composition in ex-vivo coronary specimens: A comparison with histology," Coronary Artery Disease 17(5), 425-430 (2006).

11. M. Okubo, M. Kawasaki, Y. Ishihara, U. Takeyama, S. Yasuda, T. Kubota, S. Tanaka, T. Yamaki, S. Ojio, K. Nishigaki, G. Takemura, M. Saio, T. Takami, H. Fujiwara, and S. Minatoguchi, "Tissue characterization of coronary plaques - Comparison of integrated backscatter intravascular ultrasound with virtual histology intravascular ultrasound," Circulation Journal 72(10), 1631-1639 (2008).

12. F. Alfonso, J. Dutary, M. Paulo, N. Gonzalo, M. Pérez-Vizcayno, P. Jiménez-Quevedo, J. Escaned, C. Bañuelos, R. Hernández, and C. Macaya, "Combined use of optical coherence tomography and intravascular ultrasound imaging in patients undergoing coronary interventions for stent thrombosis," Heart 98(16), 12131220 (2012).

13. L. Räber, J. H. Heo, M. D. Radu, H. M. Garcia-Garcia, G. G. Stefanini, A. Moschovitis, J. Dijkstra, H. Kelbaek, S. Windecker, and P. W. Serruys, "Offline fusion of co-registered intravascular ultrasound and frequency domain optical coherence tomography images for the analysis of human atherosclerotic plaques," EuroIntervention 8(1), 98-108 (2012).

14. R. S. C. Cobbold, Foundations of Biomedical Ultrasound, Oxford University Press, USA (2006). ISBN: 9780195168310

15. A. Ng, and J. Swanevelder, "Resolution in ultrasound imaging," Contin Educ Anaesth Crit Care Pain 11(5), 186-192 (2011).

16. A. Okamura, K. Iwakura, and K. Fujii, "ViewIT improves intravascular ultrasound-guided wiring in coronary intervention of chronic total occlusion," Catheterization and Cardiovascular Interventions 75(7), 1062-1066 (2010).

17. S. Tanaka, K. Sakamoto, R. Yamada, K. Nakagawa, P. G. Yock, P. J. Fitzgerald, F. Ikeno, and Y. Honda, "Plaque assessment with a novel high-definition 60-MHz IVUS imaging system: comparison with conventional 40MHz IVUS and Optical Coherence Tomography,” J Am Coll Cardiol 61(10_S), E1878 (2013).

18. Y. Kobayashi, H. Kitahara, S. Tanaka, K. Nakagawa, K. Okada, K. Otagiri; P. Yock, P. Fitzgerald, F. Ikeno, and Y. Honda, "TCT-363 Precision of a Novel High-Definition 60MHz IVUS in Quantitative Measurement: Comparison with Conventional 40MHz IVUS and Optical Coherence Tomography," J Am Coll Cardiol 64(11_S), B105-B106 (2014).

19. G. J. Tearney, M. E. Brezinski, S. A. Boppart, B. E. Bouma, N. Weissman, J. F. Southern, E. A. Swanson, and J. G. Fujimoto, "Catheter-based optical imaging of a human coronary artery," Circulation 94(11), 3013-3013 (1996).

20. S.-J. Park, Y.-H. Kim, S.-W. Lee, and S.-W. Park, "Left main interventions: treatment of serious potential complications," Chapter 15 (Section E) in Handbook of Complications during Percutaneous Cardiovascular Interventions, Informa UK Ltd, 211-218 (2007).

21. J. Yin, H.-C. Yang, X. Li, J. Zhang, Q. Zhou, C. Hu, K. B. Kirk Shung, and Z. Chen, "Integrated intravascular optical coherence tomography ultrasound imaging system," Journal of biomedical optics 15(1), 010512 (2010).

22. J. Yin, X. Li, J. Jing, J. Li, D. Mukai, S. Mahon, A. Edris, K. Hoang, K. Kirk Shung, M. Brenner, J. Narula, Q. Zhou, and Z. Chen, "Novel combined miniature optical coherence tomography ultrasound probe for in vivo intravascular imaging," Journal of Biomedical Optics 16(6), 060505 (2011).

23. B. H. Li, A. S. O. Leung, A. Soong, C. E. Munding, H. Lee, A. S. Thind, N. R. Munce, G. A. Wright, C. H. Rowsell, V. X. D. Yang, B. H. Strauss, F. Stuart Foster, and B. K. Courtney, "Hybrid intravascular ultrasound and optical coherence tomography catheter for imaging of coronary atherosclerosis," Catheterization and Cardiovascular Interventions 81(3), 494-507 (2013).

24. J. Li, X. Li, D. Mohar, A. Raney, J. Jing, J. Zhang, A. Johnston, S. Liang, T. Ma, K. K. Shung, S. Mahon, M. Brenner, J. Narula, Q. Zhou, P. M. Patel, and Z. Chen, "Integrated IVUS-OCT for real-time imaging of coronary atherosclerosis," JACC: Cardiovascular Imaging 7(1), 101-103 (2014).

25. X. Li, J. Li, J. Jing, T. Ma, S. Liang, J. Zhang, D. Mohar, A. Raney, S. Mahon, M. Brenner, P. Patel, K. K. Shung, Q. Zhou, and Z. Chen, "Integrated IVUS-OCT Imaging for Atherosclerotic Plaque Characterization," IEEE Journal on Selected Topics in Quantum Electronics 20(2), 6573330 (2014). 
26. J. Li, T. Ma, D. Mohar, E. Steward, M. Yu, Z. Piao, Y. He, K. K. Shung, Q. Zhou, P. M. Patel, and Z. Chen, "Ultrafast optical-ultrasonic system and miniaturized catheter for imaging and characterizing atherosclerotic plaques in vivo," Scientific Reports 5, 18406 (2015).

27. T.-H. Tsai, B. Potsaid, Y. K. Tao, V. Jayaraman, J. Jiang, P. J. S. Heim, M. F. Kraus, C. Zhou, J. Hornegger, H. Mashimo, A. E. Cable, and J. G. Fujimoto, "Ultrahigh speed endoscopic optical coherence tomography using micromotor imaging catheter and VCSEL technology," Biomedical Optics Express 4(7), 1119-1132 (2013).

28. R. Huber, M. Wojtkowski, and J. G. Fujimoto, "Fourier Domain Mode Locking (FDML): A new laser operating regime and applications for optical coherence tomography," Optics Express 14(8), 3225-3237 (2006).

29. X. Li, J. Yin, C. Hu, Q. Zhou, K. K. Shung, and Z. Chen, "High-resolution coregistered intravascular imaging with integrated ultrasound and optical coherence tomography probe,” Applied Physics Letters 97(13), 133702 (2010).

30. H.-C. Yang, J. Yin, C. Hu, J. Cannata, Q. Zhou, J. Zhang, Z. Chen, and K. K. Shung, "A dual-modality probe utilizing intravascular ultrasound and optical coherence tomography for intravascular imaging applications," IEEE Transactions on Ultrasonics, Ferroelectrics, and Frequency Control 57(12), 2839-2843 (2010).

31. J. Li, T. Ma, J. Jing, J. Zhang, P. M. Patel, K. Kirk Shung, Q. Zhou, and Z. Chen, "Miniature optical coherence tomography-ultrasound probe for automatically coregistered three-dimensional intracoronary imaging with real-time display," Journal of Biomedical Optics 18(10), 100502 (2013).

32. J. Li, J. Yin, X. Li, J. Jing, D. Mukai, S. Mahon, A. Edris, K. Hoang, K. K. Shung, M. Brenner, J. Narula, Q. Zhou, P. Patel, and Z. Chen, "Miniature integrated optical coherence tomography (OCT) - ultrasound (US) probe for intravascular imaging," Proceedings of the SPIE 8207, 82073X (2012).

33. J. Li, T. Ma, D. Mohar, A. Correa, H. Minami, J. Jing, Q. Zhou, P. M. Patel, and Z, Chen, "Diagnostic accuracy of integrated intravascular ultrasound and optical coherence tomography (IVUS-OCT) system for coronary plaque characterization," Proceedings of the SPIE 8926, 892635 (2014).

34. J. Li, H. Minami, E. Steward, T. Ma, D. Mohar, C. Robertson, K. Shung, Q. Zhou, P. Patel, and Z. Chen, "Optimal flushing agents for integrated optical and acoustic imaging systems," Journal of Biomedical Optics 20(5), 056005 (2015).

35. R. Waksman, H. Kitabata, F. Prati, M. Albertucci, and G. S. Mintz, "Intravascular ultrasound versus optical coherence tomography guidance," Journal of the American College of Cardiology 62(17 SUPPL), S32-S40 (2013).

36. H. G. Bezerra, M. A. Costa, G. Guagliumi, A. M. Rollins, and D. I. Simon, "Intracoronary Optical Coherence Tomography: A Comprehensive Review. Clinical and Research Applications," JACC: Cardiovascular Interventions 2(11), 1035-1046 (2009).

37. Y. Ozaki, H. Kitabata, H. Tsujioka, S. Hosokawa, M. Kashiwagi, K. Ishibashi, K. Komukai, T. Tanimoto, Y. Ino, S. Takarada, T. Kubo, K. Kimura, A. Tanaka, K. Hirata, M. Mizukoshi, T. Imanishi, and T. Akasaka, "Comparison of contrast media and low-molecular-weight dextran for frequency-domain optical coherence tomography," Circulation Journal 76(4), 922-927 (2012).

38. V. V. Tuchin, D. M. Zhestkov, A. N. Bashkatov, and E. A. Genina, "Theoretical study of immersion optical clearing of blood in vessels at local hemolysis," Optics Express 12(13), 2966-2971 (2004).

39. X. Xu, R. K. Wang, J. B. Elder, and V. V. Tuchin, "Effect of dextran-induced changes in refractive index and aggregation on optical properties of whole blood," Physics in Medicine and Biology 48(9), 1205-1221 (2003).

40. M. Brezinski, K. Saunders, C. Jesser, X. Li, and J. Fujimoto, "Index matching to improve optical coherence tomography imaging through blood," Circulation 103(15), 1999-2003 (2001).

41. E. A. Genina, A. N. Bashkatov, Yu. P. Sinichkin, I. Yu. Yanina, and V. V. Tuchin, "Optical clearing of biological tissues: prospects of application in medical diagnostics and phototherapy," $\mathrm{J}$ of Biomedical Photonics \& Eng 1(1), 22-58 (2015).

42. V. V. Tuchin, X. Xu, and R. K. Wang, "Dynamic optical coherence tomography in studies of optical clearing, sedimentation, and aggregation of immersed blood," Applied Optics 41(1), 258-271 (2002).

43. K. Ohtsuki, M. Hayase, K. Akashi, S. Kopiwoda, and H. W. Strauss, "Detection of monocyte chemoattractant protein-1 receptor expression in experimental atherosclerotic lesions: An autoradiographic study," Circulation 104(2), 203-208 (2001).

44. F. D. Kolodgie, A. Petrov, R. Virmani, N. Narula, J. W. Verjans, D. K. Weber, D. Hartung, N. Steinmetz, J. L. Vanderheyden, M. A. Vannan, H. K. Gold, C. P. M. Reutelingsperger, L. Hofstra, and J. Narula, "Targeting of Apoptotic Macrophages and Experimental Atheroma with Radiolabeled Annexin V: A Technique with Potential for Noninvasive Imaging of Vulnerable Plaque," Circulation 108(25), 3134-3139 (2003).

45. P. Schoenhagen, K. M. Ziada, D. G. Vince, S. E. Nissen, and E. M. Tuzcu, “Arterial remodeling and coronary artery disease: the concept of "dilated" versus "obstructive" coronary atherosclerosis," Journal of the American College of Cardiology 38(2), 297-306 (2001). 
46. G. J. Tearney, I.-K. Jang, and B. E. Bouma, "Optical coherence tomography for imaging the vulnerable plaque," Journal of Biomedical Optics 11(2), 021002 (2006).

47. C. Xu, J. M. Schmitt, S. G. Carlier, and R. Virmani, “Characterization of atherosclerosis plaques by measuring both backscattering and attenuation coefficients in optical coherence tomography," Journal of Biomedical Optics 13(3), 034003 (2008).

48. G. Van Soest, T. Goderie, E. Regar, S. Koljenović, G. L. J. H. Van Leenders, N. Gonzalo, S. Van Noorden, T. Okamura, B. E. Bouma, G. J. Tearney, J. Wolter Oosterhuis, P. W. Serruys, and A. F. W. Van Der Steen, "Atherosclerotic tissue characterization in vivo by optical coherence tomography attenuation imaging," Journal of Biomedical Optics 15(1), 011105 (2010).

49. N. Gonzalo, J. Escaned, F. Alfonso, P. Jiménez-Quevedo, B. Zakhem, C. Bañuelos, R. Hernández-Antolín, and C. Macaya, "Is refined OCT guidance of stent implantation needed?" EuroIntervention 6(Suppl G), G145-153 (2010).

50. A. V. Finn, M. Joner, G. Nakazawa, F. Kolodgie, J. Newell, M. C. John, H. K. Gold, and R. Virmani, "Pathological correlates of late drug-eluting stent thrombosis: Strut coverage as a marker of endothelialization," Circulation 115(18), 2435-2441 (2007).

51. N. Gonzalo, P. W. Serruys, T. Okamura, Z. J. Shen, H. M. Garcia-Garcia, Y. Onuma, R. J. Van Geuns, J. Ligthart, and E. Regar, "Relation between plaque type and dissections at the edges after stent implantation: An optical coherence tomography study," International Journal of Cardiology 150(2), 151-155 (2011).

52. S. Tahara, H. G. Bezerra, V. Sirbu, H. Kyono, G. Musumeci, N. Rosenthal, G. Guagliumi, and M. A. Costa, "Angiographic, IVUS and OCT evaluation of the long-term impact of coronary disease severity at the site of overlapping drug-eluting and bare metal stents: A substudy of the ODESSA trial," Heart 96(19), 1574-1578 (2010).

53. F. Alfonso, M. Paulo, N. Gonzalo, J. Dutary, P. Jimenez-Quevedo, V. Lennie, J. Escaned, C. Bauelos, R. Hernandez, and C. MacAya, "Diagnosis of spontaneous coronary artery dissection by optical coherence tomography," Journal of the American College of Cardiology 59(12), 1073-1079 (2012).

54. L. Misuraca, F. De Caro, C. Grigoratos, M. De Carlo, and A. S. Petronio, "OCT-guided stenting of a spontaneous coronary artery dissection," Cardiovascular Revascularization Medicine 13(5), 301-303 (2012).

55. K. Poon, A. Incani, A. Small, and O. C. Raffel, "Drug eluting stents trapping intramural hematoma in spontaneous coronary artery dissection and healing pattern at six months: Optical coherence tomography findings," Cardiovascular Revascularization Medicine 14(3), 183-186 (2013).

56. M. Paulo, J. Sandoval, V. Lennie, J. Dutary, M. Medina, N. Gonzalo, P. Jimenez-Quevedo, J. Escaned, C. Bañuelos, R. Hernandez, C. Macaya, and F. Alfonso, "Combined use of OCT and IVUS in spontaneous coronary artery dissection,” JACC: Cardiovascular Imaging 6(7), 830-832 (2013).

57. The Global Market for Intravascular Ultrasound Tools and Ancillary Equipment, BCC Research

58. P. R. Moreno, R. A. Lodder, K. R. Purushothaman, W. E. Charash, W. N. O'Connor, J. E. Muller, "Detection of lipid pool, thin fibrous cap, and inflammatory cells in human aortic atherosclerotic plaques by near-infrared spectroscopy," Circulation 105(8), 923-927 (2002).

59. J. Wang, Y.-J. Geng, B. Guo, T. Klima, B. N. Lal, J. T. Willerson, and W. Casscells, "Near-infrared spectroscopic characterization of human advanced atherosclerotic plaques," Journal of the American College of Cardiology 39(8), 1305-1313 (2002).

60. P. Wang, T. Ma, M. N. Slipchenko, S. Liang, J. Hui, K. K. Shung, S. Roy, M. Sturek, Q. Zhou, Z. Chen, and J.-X. Cheng, "High-speed intravascular photoacoustic imaging of lipid-laden atherosclerotic plaque enabled by a 2-kHz Barium Nitrite Raman Laser," Scientific Reports 4, 6889 (2014).

61. B. Wang, A. Karpiouk, D. Yeager, J. Amirian, S. Litovsky, R. Smalling, and S. Emelianov, "In vivo Intravascular Ultrasound-guided Photoacoustic Imaging of Lipid in Plaques Using an Animal Model of Atherosclerosis," Ultrasound in Medicine and Biology 38(12), 2098-2103 (2012).

62. Z. Piao, T. Ma, J. Li, M. Wiedmann, S. Huang, M. Yu, K. K. Shung, Q. Zhou, C. S. Kim, and Z. Chen, "High speed intravascular photoacoustic imaging with fast OPO laser at $1.7 \mu \mathrm{m}$," Applied Physics Letters 107(8), 083701 (2015).

63. W. Wei, X. Li, Q. Zhou, K. K. Shung, and Z. Chen, "Integrated ultrasound and photoacoustic probe for coregistered intravascular imaging," Journal of Biomedical Optics 16(10), 106001 (2011).

64. X. Li, W. Wei, Q. Zhou, K. K. Shung, and Z. Chen, "Intravascular photoacoustic imaging at 35 and $80 \mathrm{MHz}$," Journal of Biomedical Optics 17(10), 106005 (2012).

65. B. F. Kennedy, K. M. Kennedy, and D. D. Sampson, "A review of optical coherence elastography: Fundamentals, techniques and prospects," IEEE Journal on Selected Topics in Quantum Electronics 20(2), 6670078 (2014).

66. S. J. Kirkpatrick, R. K. Wang, and D. D. Duncan, "OCT-based elastography for large and small deformations," Optics Express 14(24), 11585-11597 (2006). 
67. J. Zhu, Y. Qu, T. Ma, R. Li, Y. Du, S. Huang, K. K. Shung, Q. Zhou, and Z. Chen, "Imaging and characterizing shear wave and shear modulus under orthogonal acoustic radiation force excitation using OCT Doppler variance method," Optics Letters 40(9), 2099-2102 (2015).

68. W. Qi, R. Li, T. Ma, J. Li, K. Kirk Shung, Q. Zhou, and Z. Chen, "Resonant acoustic radiation force optical coherence elastography," Applied Physics Letters 103(10), 103704 (2013).

69. W. Qi, R. Chen, L. Chou, G. Liu, J. Zhang, Q. Zhou, and Z. Chen, "Phase-resolved acoustic radiation force optical coherence elastography," Journal of Biomedical Optics 17(11), 110505 (2012).

70. W. Qi, R. Li, T. Ma, K. Kirk Shung, Q. Zhou, and Z. Chen, "Confocal acoustic radiation force optical coherence elastography using a ring ultrasonic transducer, ” Applied Physics Letters 104(12), 123702 (2014).

\section{Introduction}

Coronary artery disease (CAD) is the current number one cause of death worldwide [1]. CAD is ascribed to the development of atherosclerotic plaques. The vast majority of plaques remain stable after built and won't cause any acute coronary syndromes, such as heart attacks. Vulnerable (unstable) plaques, however, are prone to rupture and may lead to life-threatening events.

To detect atherosclerotic plaques, X-ray angiography is routinely employed in hospitals. By providing a two-dimensional (2D) visualization of patients' coronary arteries, angiography shows where there is a severe stenosis. However, extensive data showed that vulnerable plaques are actually less obtrusive plaques and are characterized with a large lipid core overlaid by a thin fibrous cap, which are not visible by angiography. Therefore, angiography is usually not able to accurately discern vulnerable plaques, prognose, and prevent heart attack.

A variety of three-dimensional (3D) imaging techniques have been developed to image plaques. Some techniques are non-invasive, where there is no need of inducing a catheter into body, and others are minimally invasive. Well-developed non-invasive imaging modalities include ultrasound, computed tomography (CT), magnetic resonance imaging (MRI), single photon emission computed tomography (SPECT) and positron emission tomography (PET). Ultrasound [2] (spatial resolution $\sim 400 \mu \mathrm{m}$ ) can image large superficial arteries, such as carotid, iliac and femoral arteries, by placing an ultrasound transducer closer to those arteries. CT (spatial resolution $\sim 400 \mu \mathrm{m}$ ) provides $3 \mathrm{D}$ information by rotating the X-ray source or detector around the patient's body and reconstructing acquired 2D images [3]. MRI (spatial resolution $\sim 250 \mu \mathrm{m}$ ) can characterize and quantify plaque composition by using a strong homogenous magnetic field [4]. Using a similar mechanism, magnetic resonance angiography (MRA) can assess the distribution of stenotic plaques [2]. SPECT (spatial resolution $\sim 1 \mathrm{~cm}$ ) can detect inflammations, a process closely associated with the severity of atherosclerosis, by using gamma rays [2, 4, 5]. PET (spatial resolution $\sim 4 \mathrm{~mm}$ ) can also visualize inflammation, by detecting annihilation $\mathrm{\gamma}$-photons $[2,4$, 5]. Currently, PET and SPECT are only used for preclinical study [2], unlike MRI and CT which has been used for clinical studies to image carotid arteries. However, the coronary arteries, where heart attack happens, are much smaller than carotid arteries and are constantly moving along with the cardiac and respiratory motions. At the present stage of development, none of these non-invasive imaging modalities are capable for imaging of a coronary artery with enough spatial and temporal resolution to characterize and quantify vulnerable plaques. Therefore, they are also not suitable for identifying vulnerable plaques that cause acute coronary syndromes.

On the other hand, minimally invasive methods, such as intravascular ultrasound (IVUS) and optical coherence tomography (OCT), enable clear 3D visualization of coronary arteries. IVUS is used to visualize the size of a plaque burden, such as the lipid core of a vulnerable plaque. Furthermore, postprocessing of the IVUS signal (virtual histology-IVUS and integrated backscatter-IVUS) offers another contrast to characterize plaque composition. However, the axial resolution of IVUS is only 100-200 $\mu \mathrm{m}$ and, thus, cannot measure the thickness of a fibrous cap. In contrast, the axial resolution of OCT is better than 10 $\mu \mathrm{m}$ and allows accurate measurement of the thickness of a fibrous cap. Nevertheless, OCT can only penetrate $\sim 1.5 \mathrm{~mm}$ in coronary arteries, making it challenging and unreliable for evaluating the size of a large lipid core. As shown in Table 1, OCT has high spatial resolution but low penetration depth. IVUS has deep penetration depth but low resolution. OCT and IVUS provide complementary information for high risk plaque identification.

Clinical studies, using separate IVUS and OCT catheters, also validated this observation. In 2007, a study which enrolled 30 patients with acute myocardial infarction was reported [6]. In this study, the ability of OCT and IVUS to assess vulnerable plaques was compared. In 2008, Sawada et al. [7] imaged and evaluated plaques in vivo in 56 patients using separate IVUS and OCT catheters. They found that neither modality was capable of identifying vulnerable plaques accurately, but these two modalities provided complementary information about vulnerable plaques. Accordingly, they proposed that the combined use of IVUS and OCT may be a sensible tool to detect vulnerable plaques. In 2015, an ex vivo study with a bigger sample size was conducted and validated this proposed idea [8]. In this study, 685 regions of interests were imaged. Since this study was performed on cadaver samples instead of live patients, histology slides 
Table 1 Comparison of OCT and IVUS in clinical applications in cardiology.

\begin{tabular}{|c|c|c|c|}
\hline & & OCT & IVUS \\
\hline \multicolumn{2}{|c|}{ Axial resolution } & $10 \mu \mathrm{m}$ & $100-200 \mu \mathrm{m}$ \\
\hline \multicolumn{2}{|c|}{ Penetration depth } & $1-2 \mathrm{~mm}$ & More than $5 \mathrm{~mm}$ \\
\hline \multirow{3}{*}{$\begin{array}{l}\text { Vulnerable plaques } \\
\text { identification }\end{array}$} & Detect thin fibrous cap & $\begin{array}{l}\text { Strong. The only clinically } \\
\text { available technology to } \\
\text { measure fibrous cap } \\
\text { thickness }\end{array}$ & Weak \\
\hline & $\begin{array}{l}\text { Large lipid or necrotic } \\
\text { core }\end{array}$ & $\begin{array}{c}\text { Not able to image deeper } \\
\text { than } 1.5 \mathrm{~mm}\end{array}$ & Strong \\
\hline & $\begin{array}{c}\text { Contrast of lipid and } \\
\text { others }\end{array}$ & Relatively strong & Weak \\
\hline \multirow{3}{*}{ Plaque type classification } & Calcification & Strong & Stronger than OCT \\
\hline & Lipid & Stronger than IVUS & Weak \\
\hline & Fibrosis & Stronger than IVUS & Weak \\
\hline \multirow{2}{*}{ Stent } & $\begin{array}{c}\text { Small degrees of in-stent } \\
\text { neointima }\end{array}$ & Strong & $\begin{array}{c}\text { Weak but more clinical } \\
\text { evidence reported }\end{array}$ \\
\hline & Uncovered struts & Strong & $\begin{array}{l}\text { Weak but more clinical } \\
\text { evidence reported }\end{array}$ \\
\hline
\end{tabular}

of the imaged regions could be dissected and analyzed to compare with image-guided diagnosis. By using histology as the gold standard, the positive predictive value (PPV) was found to be increased from $19 \%$ (IVUS-only) or $41 \%$ (OCT-only) to $69 \%$ when IVUS was used in combination with OCT. Thus, they concluded that the combined use of OCT and IVUS may improve the detection accuracy of vulnerable plaques. Apart from the complementary nature of IVUS and OCT in identifying vulnerable plaques, there is an increased number of studies showing that IVUS and OCT allow for assessment of different plaque compositions [9-11] and stent-tissue interaction [12], as summarized in Table 1.

It should be noted that all these studies were conducted using separate IVUS and OCT catheters and co-registered the IVUS and OCT data sets off-line. The co-registration was performed based on artery landmarks [7, 8, 13], such as side branches and calcifications, manually or semi-automatically. This process could result in human error and interobserver variances. Moreover, it cannot provide real-time evaluation of the artery and cannot fit the need for guiding intervention on the spot. An integrated IVUSOCT system can address all these issues and provide complementary information to discern vulnerable plaques simultaneously, and, thus, enable intraoperative guidance.

Therefore it can be stated that an integrated IVUS and OCT system holds great promise in improving clinical outcomes in cardiology.

Over the past 6 years, integrated IVUS-OCT systems for cardiovascular applications have been developed. In this review, the fundamentals of IVUS and OCT are described first to prepare readers about the key concerns of building an integrated IVUS-OCT system and catheter. Then the fundamental challenges and technical advances of IVUS-OCT technology are presented. In vivo and ex vivo studies assessing the potentials of IVUS-OCT for imaging plaques are also reviewed. Last but not least, some future research directions are discussed.

\section{Fundamentals of IVUS and OCT and their commercial products}

The goal of developing integrated IVUS-OCT technology is to provide the advantages of both technologies at the same time. Consequently, we will first describe fundamentals of IVUS-alone and OCTalone technologies before reviewing the integrated technology. Such an understanding is vital if IVUSOCT is to be successfully developed. In this section, important parameters and features of IVUS and OCT technologies are described, and commonly-used commercial IVUS or OCT products are introduced and compared.

There are two main components for an intravascular imaging apparatus (either an IVUS or OCT apparatus): the imaging system and the catheter. The imaging system is to generate light or ultrasound for detection and to receive and process the returned signal. The catheter is a miniaturized sensor inserted into the artery to acquire signals in situ.

\subsection{IVUS}

The most important parameters of an IVUS apparatus are the resolution of the system and the outer diameter of the catheter.

The axial resolution of IVUS is determined by the central frequency and the bandwidth of the ultrasound system $[14,15]$ :

$$
B=\frac{V}{2 \cdot f_{o} \cdot B W}
$$

where $f_{0}$ is central frequency, $\mathrm{BW}$ is the fractional frequency bandwidth, and $\mathrm{V}$ is speed of sound. The comparison of central frequencies of commercial IVUS 
Table 2 Comparison of commercial IVUS products.

\begin{tabular}{|c|c|c|c|c|}
\hline $\begin{array}{l}\text { Company } \\
\text { name }\end{array}$ & Product name & $\begin{array}{l}\text { Central } \\
\text { frequency } \\
(\mathrm{MHz})\end{array}$ & $\begin{array}{l}\text { Outer } \\
\text { diameter } \\
\text { (French) }\end{array}$ & Other features \\
\hline \multirow{3}{*}{$\begin{array}{l}\text { Boston } \\
\text { scientific }\end{array}$} & iCross $^{\mathrm{TM}}$ & 40 & 3.5 & \\
\hline & $\begin{array}{l}\text { Atlantis }^{\mathrm{TM}} \mathrm{SR} \\
\text { Pro }\end{array}$ & 40 & 3.6 & \\
\hline & OptiCross $^{\mathrm{TM}}$ & 40 & 3.15 & $\begin{array}{l}\text { It performs a higher axial resolution than those of other } \\
\text { Boston Scientific IVUS products. }\end{array}$ \\
\hline \multirow{2}{*}{$\begin{array}{c}\text { Philips } \\
\text { (Volcano) }\end{array}$} & Revolution $\mathbb{R}$ & 45 & 3.2 & Virtual histology function is available. \\
\hline & Eagle Eye ${ }^{\circledR}$ & 20 & 3.5 & $\begin{array}{c}\text { The sensor is a phase array and ChromaFlo } \AA \text { imaging } \\
\text { function is available. }\end{array}$ \\
\hline \multirow{2}{*}{ Terumo } & View IT® & 40 & $2.6(3.2)^{*}$ & $\begin{array}{l}\text { It has a smaller diameter of its imaging window (2.6 } \\
\text { French) than its proximal shaft ( } 3.2 \text { French). }\end{array}$ \\
\hline & Intrafocus ${ }^{\circledR}$ & 40 & $2.4(3.2)$ & $\begin{array}{l}\text { Two guide-wire channels: at the bent portion, more } \\
\text { stable }\end{array}$ \\
\hline ACIST & $\begin{array}{l}\text { Kodama }{ }^{R} \\
\text { HD-IVUS }\end{array}$ & 60 & N.A. & $\begin{array}{l}\text { There are reports }[17,18] \text { that this product can run at a } \\
\text { faster imaging speed than other commercial IVUS } \\
\text { products, but no product is available yet for sale. } * *\end{array}$ \\
\hline
\end{tabular}

products is summarized in column 3, Table 2. Two commercial products, Opticross and iCross from Boston Scientific, have the same center frequency, but Opticross performs with a superior resolution because of its broad bandwidth.

The lateral resolution of IVUS is determined by the beam spot exiting the ultrasound transducer $[14,15]$ :

$$
R=\frac{V}{f_{0}} \cdot F \#,
$$

where $F \#$ is the quotient of the focal length $f$ and the outer diameter $\mathrm{D}$ of the transducer aperture.

Lateral resolution is not discussed as often as the axial resolution because most intravascular applications focus on resolving the cross-sectional features of the multilayered structure of the artery lumen, which are in the axial direction.

The outer diameter over the entire length of a catheter is usually not a constant value. The crossing profile, i.e., the outer diameter of the distal tip, determines how small a vascular lesion that this catheter can go into, while the proximal shaft size determines the size of the guiding catheter that this imaging catheter can be used with and pass through. For example, a key feature of Terumo's IVUS products is the small crossing profile although the proximal shaft diameters of these products are similar to other companies' products. This special design is used for accessing arteries with large plaque burdens, such as those with chronic total occlusion [16]. The comparison of outer diameters of commercial IVUS products is summarized in column 4, Table 2 .

Some other features of IVUS commercial products, which are possible to be added in an integrated IVUSOCT apparatus, are also listed in Table 2. Volcano is a company dedicated to developing IVUS technology. It has developed and implemented a number of software based imaging processing features, such as virtual histology, which provides automatic analysis of plaque components, and ChromaFlo $\AA$, which indicates the locations of blood vessels, to enhance IVUS capability. Recently, Volcano was acquired by Philips. Silicon Valley Medical Instrument, a relatively new player in the IVUS market, was acquired by ACIST. Its product is highlighted by a higher frequency and higher imaging speed than those of established IVUS players.

\section{$2.2 O C T$}

Unlike IVUS, which was invented 60 years ago, OCT is relatively new, and the first use of OCT in cardiology was briefly reported in Circulation in 1996 [19]. Two decades of research in OCT cardiovascular imaging has led to the generation of two commercial product-lines, from St. Jude Medical and Terumo.

The two most important features, the outer diameter of the catheter and the imaging speed of commercial OCT products, are compared in Table 3. Similar to IVUS, the outer diameter of a catheter determines whether a catheter can cross a heavily blocked coronary artery. There are several generations of St. Jude Medical products: the imaging speed has increased from 20 frames per second (fps, M2/M3) to $100 \mathrm{fps}$ (C7XR) and now to 180 fps (ILUMIEN ${ }^{\text {TM }}$ OPTISTM). However, they are very similar in terms of the catheter size and thus only the latest product is listed here for comparison.

Some other clinically relevant features, which could be considered in future to improve the design of an IVUS-OCT catheter, are also listed. Purging, injecting saline into the lumen of a catheter, is needed for St. Jude Medical products. This extra step is to reduce astigmatism and high reflection signals caused by the catheter sheath and to decrease non-uniform rotational distortion (NURD). But some clinicians may find this step cumbersome and prefer using a non-purge catheter. 
Table 3 Comparison of commercial OCT products.

\begin{tabular}{ccccc}
\hline & $\begin{array}{c}\text { Crossing } \\
\text { profile }\end{array}$ & Speed & Other feature \\
\hline Terumo & $\begin{array}{c}\text { LUNAWAVETM } \\
\text { OFDI }\end{array}$ & $2.4 \mathrm{~F}$ & $160 \mathrm{fps}$ & No purge is needed. \\
\hline $\begin{array}{c}\text { St. Jude Medical } \\
\text { (Lightlab) }\end{array}$ & $\begin{array}{c}\text { ILUMIENTM } \\
\text { OPTIS }\end{array}$ & $2.7 \mathrm{~F}$ & $180 \mathrm{fps}$ & $\begin{array}{c}\text { It has a dual-lumen tip design for } \\
\text { excellent handling and automated, } \\
\text { continuous calibration. }\end{array}$ \\
\hline
\end{tabular}

As shared by both IVUS-alone and OCT-alone technologies, the speed, the catheter size, and the image quality (such as spatial resolution) are key features. Although it is ideal to keep the best performance of each system when integrating IVUS and OCT together, there are many theoretical and engineering challenges in achieving this goal. Technical advances addressing these challenges, in terms of the system and the catheter, will be separately described in the following two sections.

\section{Integrated IVUS-OCT system}

The foremost challenge of a clinically-adaptable integrated IVUS-OCT system lies in enabling high imaging speed [13]. There is a large gap between the imaging speeds of current commercial IVUS and OCT systems. For safe intracoronary imaging in humans, an ultrafast integrated IVUS-OCT imaging system, which can image at over 70 fps and take less than 4 seconds for imaging a coronary artery, would be critical to translate this technology to clinical applications. By using an ultrafast system, the risk of catheter-induced spasm [20] and the use of a toxic contrast agent would be significantly reduced. This breakthrough could expedite the translation of the IVUS-OCT technology into clinical practice. However, the speed of most stateof-the-art commercial IVUS systems is only around 30 fps. No IVUS system, either commercial or investigational, running over $70 \mathrm{fps}$ has been reported. Thus, to develop an ultrafast IVUS-OCT system, we have to first develop an ultrafast IVUS sub-system and then build a synchronized IVUS-OCT system without sacrificing the imaging quality of either sub-system.

A common design for an integrated IVUS-OCT system is shown in Fig. 1. IVUS and OCT sub-systems are used getting IVUS and OCT signals, respectively. Acquired IVUS and OCT signals are then sent to a data acquisition (DAQ) card and work station for processing. Radial scanning is usually achieved by using a motor to rotate the optical rotary joint and, hence, the probe. The electrical and optical signals are transferred from a rotational to a stationary side usually by an electrical slip ring and an optical rotary joint, respectively. The pullback movement of a catheter is induced by using a linear stage. The imaging speed of the system (i.e., the rotation speed of an imaging probe) is usually determined by the system. However, the transmission of rotation, mechanical strength and coaxial of the catheter may cause non-uniform rotational distortion (NURD) during high speed rotation and thus limit the rotation speed of an OCT probe.

Previous research on different generations of IVUSOCT systems can be summarized as a journey to increase the imaging speed of this common system design. In 2010, a system with a speed of 1 fps was reported [21]. By using a digitizer for data acquisition and a graphics processing unit (GPU) for signal processing, 4 fps was reached in 2011 [22]. A 5-fps imaging system, which used separated data acquisition boards for IVUS and OCT signals, was published in 2013 [23]. Soon after this, $20 \mathrm{fps}$, a speed that is the same order of that of a commercial IVUS system, was achieved and safely demonstrated in vivo in pigs and rabbits [24, 25]. In 2015, an integrated IVUS-OCT system running at an imaging speed of $72 \mathrm{fps}$ was reported [26] by using a customized slip ring, an ultrahigh write-speed solid-state drive (SSD), and an ultrasound pulser/receiver faster than those employed in a commercial IVUS system [4]. This breakthrough facilitates the implementation of IVUS-OCT into clinical practice. In the following paragraphs, key elements and difficulties of previously-reported IVUSOCT systems will be summarized.

\subsection{OCT sub-system}

In an integrated IVUS-OCT system, Fourier Domain OCT (FDOCT) is usually used. FDOCT is achieved by calculating the Fourier transform of an acquired spectra. Mechanically scanning the reference arm is not needed to get depth information; thus, a faster imaging speed can be achieved using FDOCT instead of time domain OCT (e.g., the first generation commercial system St. Jude Medical M2/M3). The interference signal in the frequency domain (i.e., wavelength domain) is acquired using either a frequency-swept laser source (swept source OCT, SSOCT) or a spectrometer using a high speed line-scan camera as the detector (Spectral domain OCT, SDOCT). With the development of fast swept lasers [27, 28], the SSOCT is able to scan at a few megahertz. In general, the SSOCT system is also more compact than SDOCT. Accordingly, a SSOCT system was commonly used to be integrated with an IVUS subsystem for dual-modality intravascular imaging.

\subsection{IVUS sub-system}

The IVUS sub-system is achieved by using an IVUS pulser/receiver, which can be bought as one instrument or built by using a separate pulser [23], a 

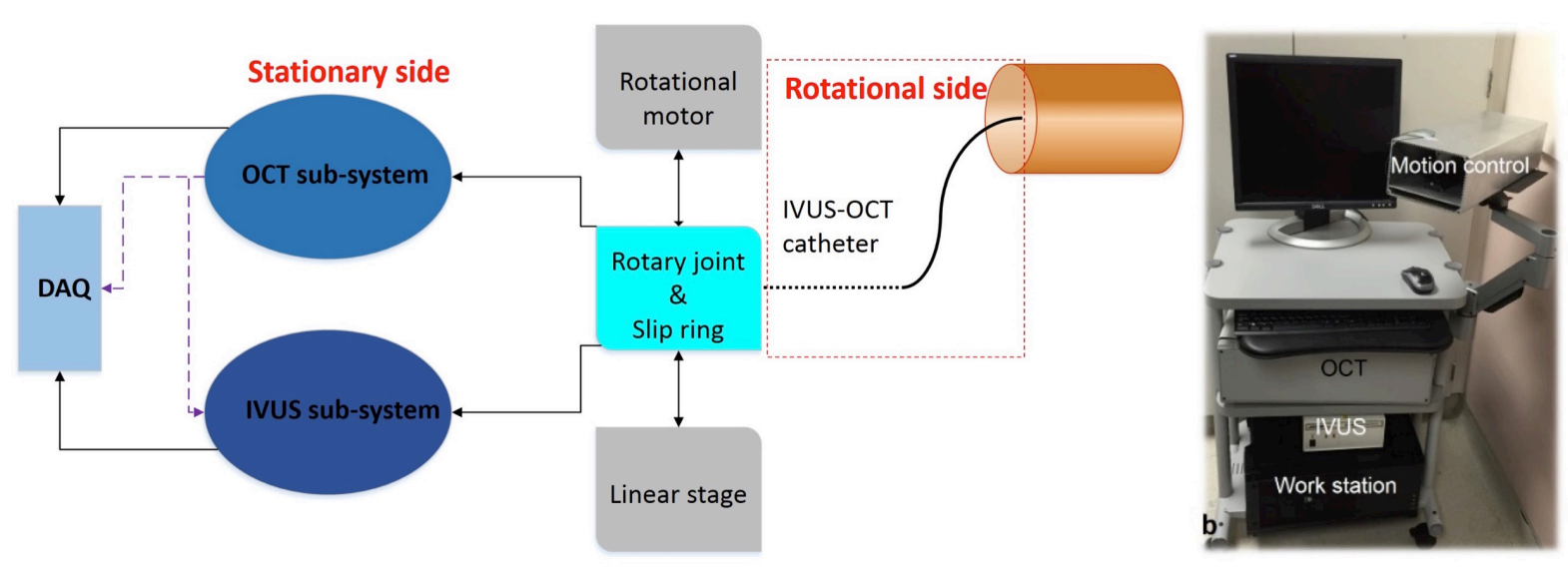

Fig. 1 Diagram (a) and photo (b) of a fully-integrated IVUS-OCT system. Black solid lines indicate IVUS and OCT signal transfer paths. Purpule dash lines denote the trigger signals.

transmit/receive switch, and a pre-amplifier. A pulse energy of $1-5 \mu \mathrm{J}$ is usually used to drive an IVUS transducer. In one design of the IVUS-OCT systems [21-23, 29], a Panametrics PR5900 pulser/receiver (Olympus NDT, Kennewick, WA) was used to drive the transducer for pulse generation and subsequent echo signal detection. For ultra-high speed imaging, a 50 kHz-external-triggered ultrasound pulser (PR-H4, JSR Ultrasonics, Pittsford, NY) [26] and a customized receiver were used to drive the transducer and detect the eco signal, respectively.

\subsection{Synchronization of the system}

Synchronization between the OCT sub-system, the IVUS sub-system and the DAQ card in the integrated system design is critical. Otherwise, the IVUS imaged location may not match that of OCT. Usually the A-line trigger signal of the OCT sub-system, which indicates the beginning of an OCT acquisition, is sent to the IVUS sub-system and the DAQ card for synchronizing the entire integrated system, see purple dash lines in Fig. 1. There could be other ways of synchronization, such as altering timing between IVUS-OCT by using a function generator, or acquiring multiple OCT frames while only acquiring a single IVUS frame (due to IVUS's low imaging speed).

\subsection{The fundamental challenge for an IVUS- OCT system}

Because it takes time for a sound wave to travel, there is a theoretical imaging-speed limit of an IVUS-OCT system. For the IVUS sub-system, when the rotation speed is over a certain value, the echo signal generated by the sample cannot be detected by the aperture of the IVUS transducer. This is because the transducer had already rotated to a different location and there is little overlap between the new location and the original location, see Fig. 2. A simple calculation can be used to estimate the theoretical limitation of IVUS sub-system.
If we want to image to $5 \mathrm{~mm}$ deep, $\mathrm{t} 1$, which represents the time of the ultrasound wave to travel from the transducer to the sample and back to the transducer, is $10 \mathrm{~mm} / 1560 \mathrm{~m} / \mathrm{s}=6.4 \mu \mathrm{s}$. If a $50 \%$ overlap is needed to maintain enough detection sensitivity, $\mathrm{t} 2$, the time taken to rotate between 2 frames, needs to be considerably bigger than $\mathrm{t} 1$. Thus, the maximal speed of this system is calculated to be $78 \mathrm{kHz}$. Assume acquiring $500 \mathrm{~A}$ lines per frame, the theoretical limit for IVUS is 156 frames per second. In addition, the less overlap that there is between the end and original locations and the farther that the sound travels (i.e., imaging a deeper sample), the less signal that can be collected. Accordingly, the sensitivity of an IVUS system drops when running at an ultrafast speed. We tested the contrast to noise ratio (CNR), an important indicator of the IVUS system sensitivity, at different imaging speeds. It was found that CNR was gradually decreasing as the speed of the system increased: $\mathrm{CNR}=6.49 \mathrm{~dB}$ (at $25 \mathrm{fps}$ ); $\mathrm{CNR}=6.19 \mathrm{~dB}$ (at $50 \mathrm{fps}$ ) and $\mathrm{CNR}=6.18 \mathrm{~dB}$ (at $72 \mathrm{fps}$ ). This experiment finding validated our theoretical analysis. On the other hand, there is almost no theoretical limit for OCT imaging speed since the speed of light is significantly higher $(\sim 20,000$ times in blood) than that of sound.

\subsubsection{OCT sub-system and IVUS sub-system}

For the OCT sub-system, a $50 \mathrm{kHz}$ (or higher frequency) swept laser is commercially available. However, off-the-shelf pulser/receivers, which fit the requirements of SNR, pulse power, external trigger compatibility, and frequency response for an IVUS subsystem, are usually running at a speed below $30 \mathrm{kHz}$. Thus, a customized or home-made pulser/receiver is needed for an ultrafast imaging system.

\subsubsection{Design of rotary system}

Similar to the ultrafast pulser/receiver, there are few offthe-shelf slip rings that can transmit a signal over 50 
fps, while maintaining a decent performance. To keep an OCT and IVUS imaging probe co-axial even at the proximal end, a through-hole slip ring is usually used. The imaging probe is inserted through the hole of the slip ring. A 72 fps IVUS-OCT system was reported recently where a customized slip ring was used [26].

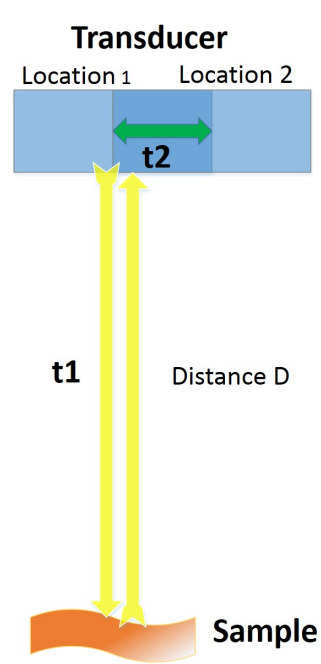

Fig. 2 Demonstration of the theoretical speed limit of an IVUS system.

\subsubsection{IT challenge}

Ultrafast imaging also requires the DAQ card and the work station to transfer, process and display the signal at an ultrahigh speed. GPU, a PCI express bus for transferring the data and a high writing speed SSD can be used to address this challenge. To transfer and save the data at an ultrahigh speed, a SSD with a write/read/transfer speed of over $800 \mathrm{Mbits} / \mathrm{s}$ is needed: if 8192 points are acquired in each A-line for both two 12-bit channels at $50 \mathrm{kHz}$ A-line speed, there will be 819.2 Megabits data coming into the SSD every second. To process this large amount of data, an advanced GPU is needed. A thorough analysis of the requirement of a GPU was described by $\mathrm{Li}$ et al. [25]. The speed of commercially available GPU has increased dramatically in the past five years, and this fits the need of an ultrafast IVUS-OCT system.

\section{Catheter designs}

Another important component of an intravascular imaging apparatus is its imaging catheter. An intravascular imaging catheter usually consists of an imaging core (probe) and an outer catheter sheath. At the tip of the imaging core, both OCT and IVUS imaging sensors are located. The catheter sheath is used outside the imaging core to avoid cross-contamination between the imaging core and the biomedical tissue. In order to avoid any catheter-induced injury to an artery and catheter-induced spasm, the outer diameter of such an imaging catheter is suggested to be smaller than 1.2 $\mathrm{mm}$. Consequently, the major challenge of catheter design is to fabricate the catheter small enough and adaptable for safe routine clinical procedures while maintaining good OCT and IVUS imaging qualities.

\subsection{Probe design}

An integrated probe is made by assembling OCT and IVUS sub-probes. An OCT sub-probe can use either a GRIN lens, GRIN fiber or ball lens design. Either a single crystal square transducer or a ring transducer, with a center frequency at $35 \mathrm{MHz}$ to $45 \mathrm{MHz}$, can serve as an IVUS sub-probe.

The foremost difficulty in making an intracoronary catheter is to make it small while maintaining its imaging quality. Usually, we want to make the IVUS and OCT sub-probes as small as possible. However, there is a trade-off between the lateral resolution and the size of a probe. If the depth of focus (DOF) is kept constant, the smaller the aperture size, the worse the lateral resolution. Thus, an optical aperture larger than $0.3 \mathrm{~mm}$ is commonly applied to maintain high lateral resolution and large DOF. Previously reported generations of IVUS-OCT probe designs address this issue by using different configurations and methods to minimize the IVUS or OCT sub-probes while keeping imaging quality, see Table 4.

The first demonstration of an integrated IVUS-OCT probe (see Gen 1.1 in Fig. 3) was reported in 2010 [21]. This probe used a side-by-side arrangement of IVUS and OCT sensors. A different side-by-side design which employed a common mirror to reflect the sound wave and light beam (see Gen 1.2 in Fig. 3) was published soon after this one [30]. Subsequently, a co-axial probe design (see Gen 2 in Fig. 3) was reported in which a ring IVUS transducer was used, and an OCT sub-probe was inserted inside the ring transducer [29].

The first IVUS-OCT probe capable of imaging in a coronary artery in vivo was demonstrated in 2011, employing a sequential configuration [22] (see Gen 3 in Fig. 3). This design enabled a smaller outer diameter than the side-by-side designs [21, 30], while using the same IVUS and OCT sub-probes and thus keeping similar imaging quality. Notably, this probe had the same outer diameter as a commercial IVUS-only probe. In 2012, a probe design (see Gen 4 in Fig. 3) very similar to the original side-by-side design was reported [23]. However, in this design, a much smaller OCT probe $(0.14 \mathrm{~mm}$ GRIN lens) was used, and there was a 90-degree difference between the exiting angles of the light beam and the sound wave.

As a matter of fact, not only is a small outer diameter important to ensure the safety of using a catheter in a coronary artery, the length of the probe rigid-part is also critical for safe operations. There are many sharp turns in the path of inserting a catheter from a femoral artery to an aorta and to a coronary artery. A probe with a short rigid-part length can be flexible and easily navigated into a coronary artery. The most recently published back-to-back design [31] (see Gen 5 in Fig. 3) addressed the strict size requirements of OD and rigid-part length at the same time. Compared with previous designs, the length of the OCT sub-probe was 


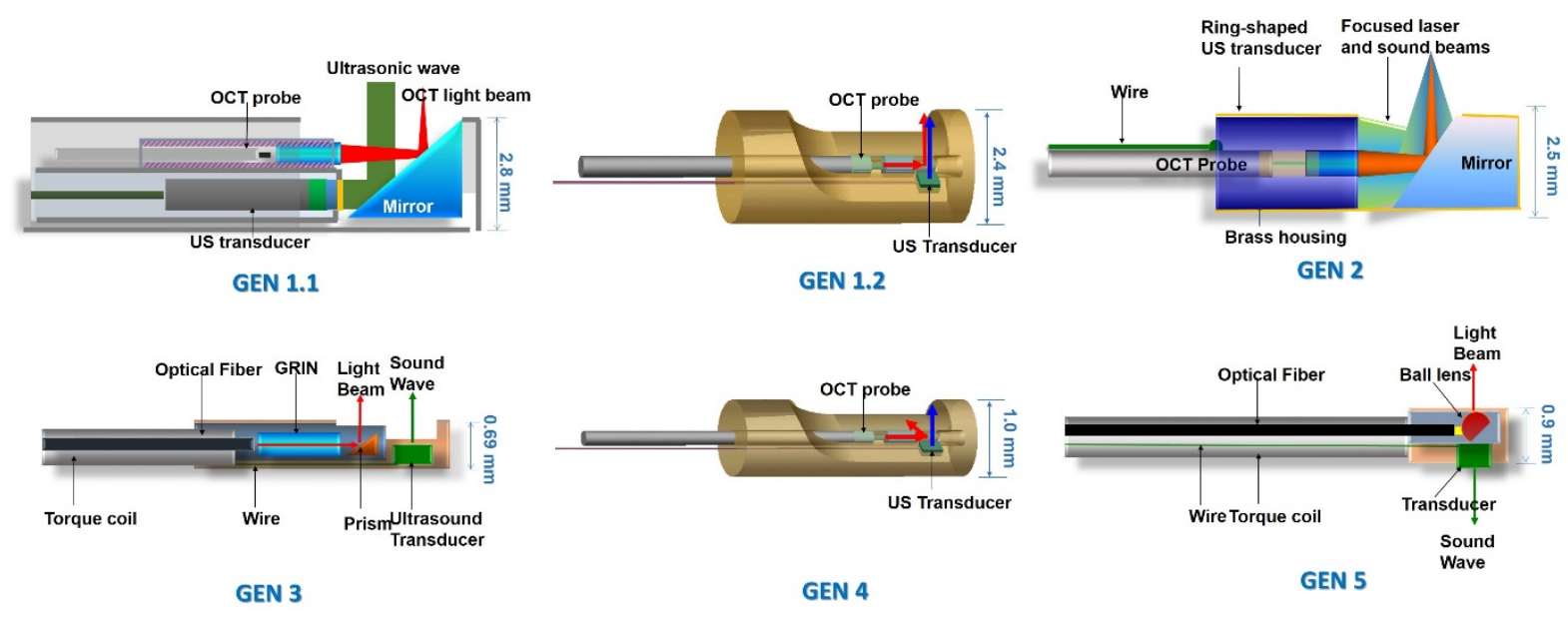

Fig. 3 Different generations of IVUS-OCT probes.

Table 4 Comparison of different generations of IVUS-OCT probes.

\begin{tabular}{cccccc}
\hline & Configuration & OD & $\begin{array}{c}\text { Estimated } \\
\text { rigid-part length }\end{array}$ & $\begin{array}{c}\text { Size of the IVUS } \\
\text { sub probe }\end{array}$ & $\begin{array}{c}\text { Size of the OCT } \\
\text { sub probe }\end{array}$ \\
\hline Gen $1.1[21]$ & Side by side & $2.4 \mathrm{~mm}$ & $5 \mathrm{~mm}$ & $0.4 \mathrm{~mm}$ by $0.4 \mathrm{~mm}$ & $0.5 \mathrm{~mm}$ GRIN \\
\hline Gen $1.2[30]$ & Side by side & $2.8 \mathrm{~mm}$ & $5 \mathrm{~mm}$ & $0.4 \mathrm{~mm}$ by $0.4 \mathrm{~mm}$ & $0.5 \mathrm{~mm}$ GRIN \\
\hline Gen $2[29]$ & Ring & $2.4 \mathrm{~mm}$ & $5.5 \mathrm{~mm}$ & $2 \mathrm{~mm}$ by $5.5 \mathrm{~mm}$ & $0.7 \mathrm{~mm}$ GRIN \\
\hline Gen $3[22]$ & Sequential & $0.69 \mathrm{~mm}$ & $4 \mathrm{~mm}$ & $0.4 \mathrm{~mm}$ by $0.4 \mathrm{~mm}$ & $0.35 \mathrm{~mm}$ GRIN \\
\hline Gen $4[23]$ & Side by side & $0.9 \mathrm{~mm}$ & $2.5 \mathrm{~mm}$ & $0.5 \mathrm{~mm}$ by $0.5 \mathrm{~mm}$ & $0.14 \mathrm{~mm}$ GRIN \\
\hline Gen $5[31]$ & Back to back & $0.9 \mathrm{~mm}$ & $1.5 \mathrm{~mm}$ & $0.4 \mathrm{~mm}$ by $0.4 \mathrm{~mm}$ & $0.3 \mathrm{~mm}$ ball lens \\
\hline
\end{tabular}

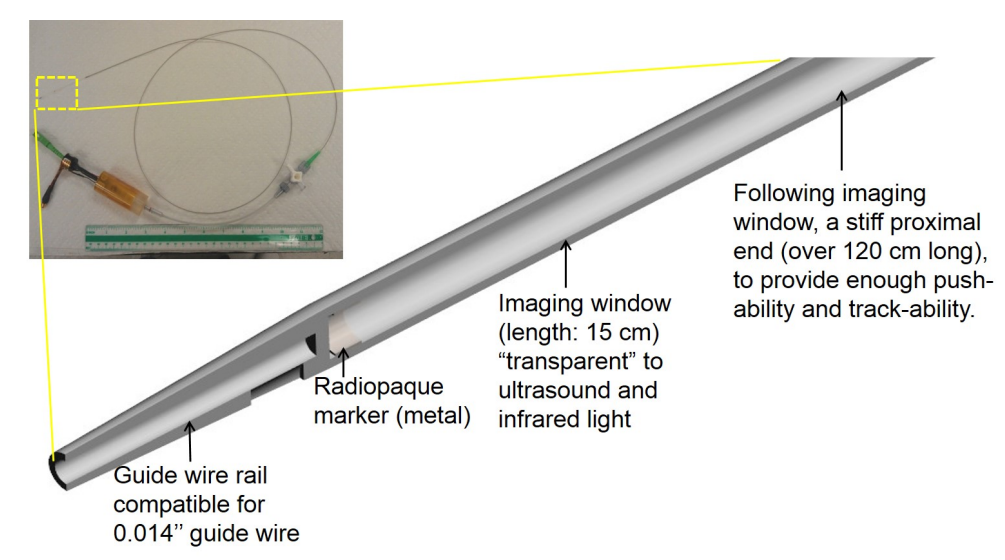

Fig. 4 Requirements of an IVUS-OCT outer sheath.

reduced by using a ball-lens design instead of a traditional GRIN lens design. The thickness of the IVUS probe was reduced by connecting the center core of a coaxial cable to the side of the backing rather than to its back surface. Although rigid-part lengths of previous generations were not reported, approximate numbers, estimated according to the sizes of IVUS and OCT sensors and published figures, are listed in Table 4 for comparison.

It is important to note that both the Gen 2 ringtransducer design [29] and Gen 5 back-to-back design [31] provide true co-axial IVUS-OCT imaging, which is not achieved by other designs.

\subsection{Outer catheter sheath design}

Essential considerations in making an IVUS-OCT outer catheter sheath [32] are listed in Fig.4.

It is critical to select optimal materials for the design of an outer catheter sheath. Usually, the higher the mechanical strength (thicker material or material with a higher durometer), the higher the reflection that will begenerated. To ensure the high pushability of the catheter and the high transparency of the catheter's imaging window, we used different materials for the imaging window and the proximal side of the catheter. We used a high durometer material for the proximal side to provide high pushability and trackability. A 
relatively soft, low-ultrasound-attenuation material was used to reduce light and sound attenuations. These two materials were thermally bonded together to ensure a smooth transition and a strong connection between the imaging window and the proximal side.

When performing IVUS-OCT imaging in vivo, an $\mathrm{X}$-ray $\mathrm{C}$-arm and a guiding wire are used to position it smoothly into a coronary artery. Thus, radiopaque markers, a guide wire rail and a telescope design, which enable pull-back of the catheter and avoid kinking during movements, are also needed in the outer catheter sheath.

\section{In vivo validation}

After an imaging apparatus are successfully built, in vivo animal studies are needed to be conducted, so as to validate the safety and effectiveness of the design. The first in vivo demonstration of integrated IVUS-OCT was reported in 2011 by using a healthy rabbit aorta [22]. In 2014, studies showing the ability of imaging plaques and imaging coronary arteries in vivo were published $[24,25]$. Recently, we reported ultrafast speed IVUSOCT for in vivo imaging [26].

One common challenge for all in vivo experiments is the strong attenuation introduced by intraluminal blood. This doesn't pose a problem for ex vivo experiments $[24,26,31,33]$ summarized in the next section, since blood was usually drained from cadaver samples before imaging. Prior to in vivo imaging, an optical flushing agent is needed to be selected [34], so as to acquire clear IVUS and OCT images simultaneously, without inducing a great amount of toxicity. Since X-ray contrast agents (such as iohexol and iodixanol) are often used in patients in both IVUS and OCT procedures [35, 36], they were predicted as effective IVUS-OCT flushing agents and were commonly used in in vivo IVUS-OCT experiments [24-26]. However, alternative flushing agents, which have less side-effects than X-ray contrast agents and better OCT or IVUS clearing effects [34, 37-41], can be used in vivo experiments in future. Potential alternative flushing agents include mannitol, dextran, glucose solution and propylene glycol [42]. A high concentration of dextran was demonstrated to be useful for simultaneous IVUS-OCT imaging and could replace unsafe contrast agents [34].

A rabbit model is one of the most frequently used and successful animal models for atherosclerosis research [43]. Lesions similar to atherosclerotic plaques can be developed in the abdominal aortas of rabbits [44], where the size of artery lumen is similar to the human coronary artery lumen. A smaller animal model such as a rat cannot be utilized given the small size of its vessels and heart. Representative IVUS-OCT image pairs obtained in an atherosclerotic rabbit in vivo are shown in Fig. 5. Intimal thickening (see arrows in Fig. 5) can be observed by IVUS and OCT images.

Swine is another commonly used animal model for cardiovascular research. Porcine models are regularly used prior to human cardiovascular research to validate the mechanical properties (pull, torsion, flex, buckling, pull back, resistance, leaking, etc.) and clinical adaptability of catheters. In general, the cost of a pig is lower than that of a dog or primate. Pigs also have better facility acceptance and anatomical similarities than dogs. For cardiovascular research, pigs are one of the best animal models. The key reasons are: 1) a pig has a coronary artery distribution more similar to a human than other animals; 2) a pig has similar lipoproteins (critical molecules related to the atherosclerosis process) as humans; 3) the morphology and biochemistry of pig plaque is similar to that of human plaque [45]. Two representative IVUS-OCT image pairs obtained in healthy swine in vivo are shown in Fig. 6. In Fig. 6 Ia, an OCT image illustrates the three-layer structure of the artery wall. From the center of the OCT image, there is a high-signal thin band corresponding to the intima, followed by a high-signal strip corresponding to the external elastic lamina (EEL), and finally a low-signal area corresponding to the adventitia. Since this swine artery is healthy, there is no positive vascular remodeling. OCT is able to penetrate all three layers of the artery wall and provide a clear visualization of layers. On the contrary, the layer structure in an artery with a lipid-rich plaque (see Fig. 7) is sometimes difficult to visualize with OCT because lipid is a high attenuation source for an optical signal. In the IVUS image (Fig. $6 \mathrm{Ib}$ ), the three-layer structure of the swine coronary artery (wall thickness, $0.3 \mathrm{~mm}$ ) is hardly visualized with a resolution of $60 \mu \mathrm{m}$. In Figs. 6 Ia and $\mathrm{Ib}$, LAD branching can be visualized.

These animal studies validated that integrated IVUSOCT is a feasible and safe technique for in vivo visualization of atherosclerotic plaques with high resolution and deep penetration simultaneously.

\section{Clinical applications of IVUS-OCT in cardiology}

IVUS-OCT has great potential for improving clinical management of cardiovascular diseases. In this section, applications where IVUS-OCT could be used to improve on current procedures will be summarized.

\subsection{Classification of different plaque types}

In 2006, three studies were published assessing the accuracy of IVUS or OCT using separate IVUS and OCT catheters. They all reached a similar conclusion that IVUS or OCT shows different strengths for tissue characterization of plaques, as summarized in Table 1. With the development of IVUS-OCT, the ability to classify different plaque types has been greatly improved. Figure 7 shows examples of IVUS-OCT image pairs of plaques with different compositions. In the IVUS image (Fig. 7 Ia, Fig. 7 IIa and Fig. 7 IIIa), positive vascular remodeling, i.e., intimal thickening, is identified by arrows. The total plaque volume and threelayer structure of the artery wall can also be visualized. Acoustic shadow in Fig. 7 IIa shows the location of a calcified plaque. However, it is difficult to classify the plaque types of Fig. 7 Ia and 7 IIIa from the IVUS 


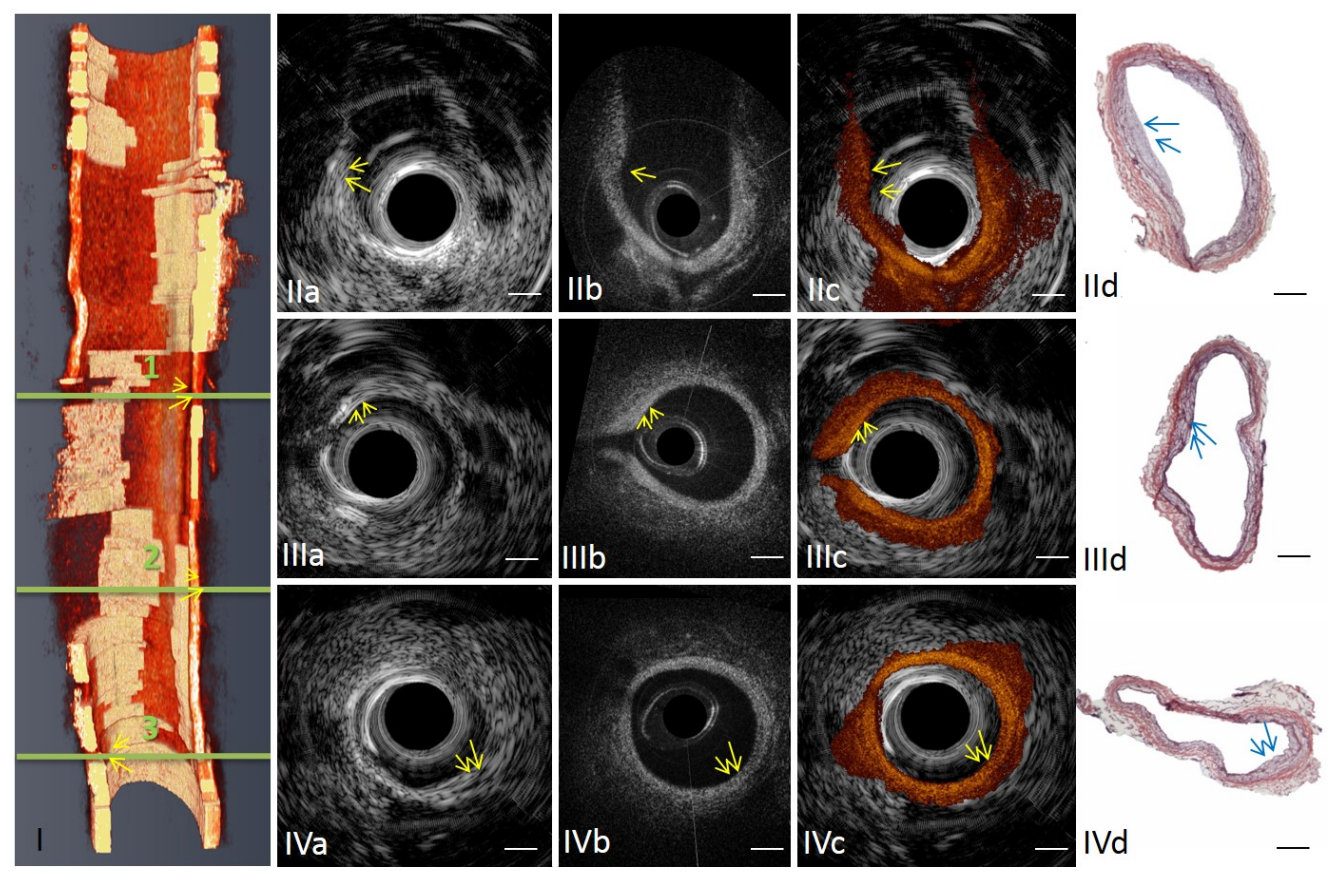

Fig. 5 IVUS-OCT imaging of a rabbit abdominal aorta in vivo. (I) Three-dimensional cut-away rendering of the volumetric data set acquired with an intravascular catheter in abdominal aorta of a live rabbit. The volume comprises 288 frames of images acquired in $4 \mathrm{~s}$ during the injection of iohexol at a rate of $3 \mathrm{ml} / \mathrm{s}$. Red, artery wall; semitransparent white, lipid. Circular cross-section IVUS (IIa) (IIIa) (IVa) OCT (IIb) (IIIb) (IVb) fused IVUS-OCT (IIc) (IIIc) (IVc) image pairs and the corresponding H\&E histology photos (IId) (IIId) (IVd) at locations 1, 2 and 3 denoted in (I). Arrows point at lipid-rich plaque regions. Scale bar: $0.5 \mathrm{~mm}$. The shape of this artery changed between in vivo imaging and histology due to the reduced intra-lumen pressure after this artery was harvested. Reprinted from [26], with permission.
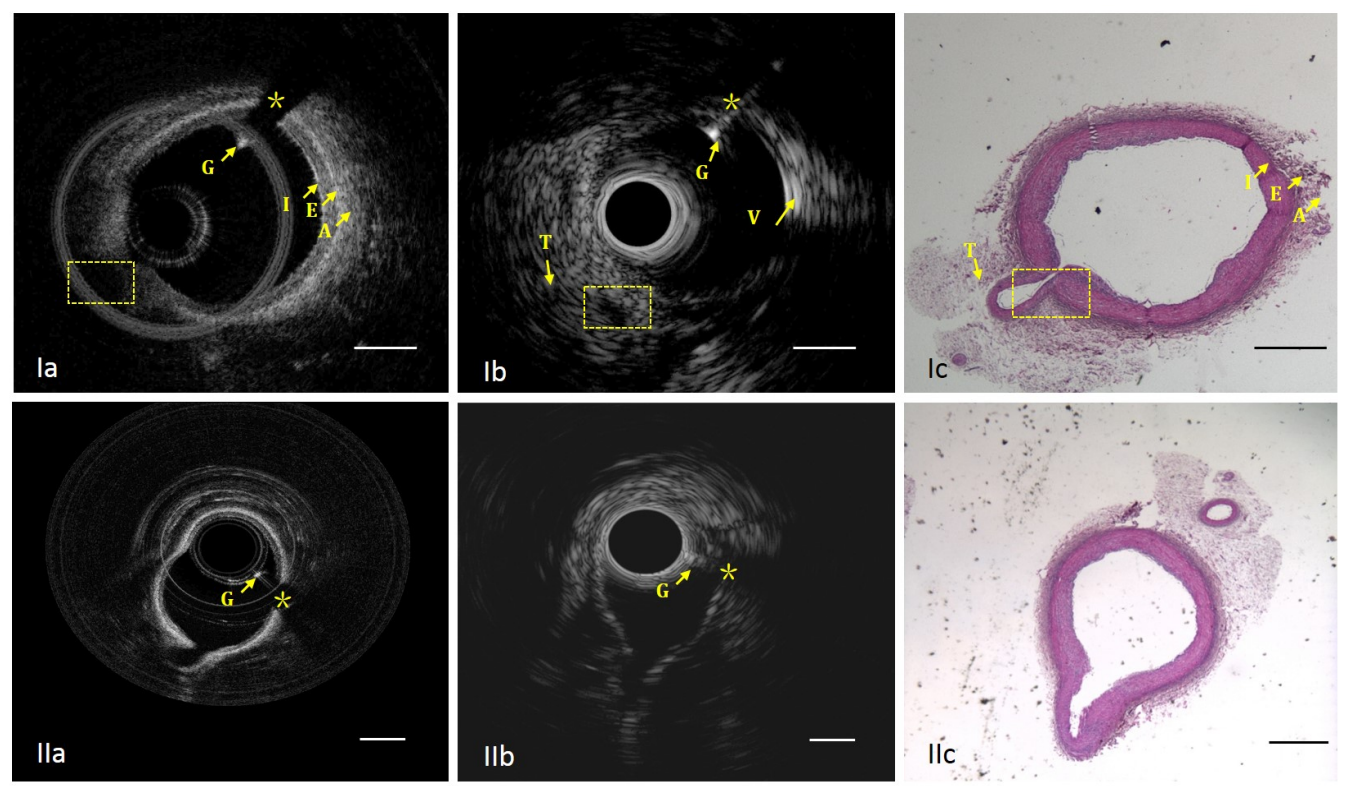

Fig. 6 An IVUS-OCT image pair obtained in a normal swine coronary artery in vivo during the injection of iohexol. (Ia) OCT image (Ib) IVUS image (Ic) corresponding H\&E histology. Another IVUS-OCT image pair, also obtained in swine, demonstrates LAD branching. (IIa) OCT (IIb) IVUS cross-sectional image. (IIc) Corresponding H\&E histology. * denotes guidewire artifact. G: guide wire. T: tissue. V: vessel. I: intima. E: EEL. A: adventitia. Yellow boxes denote LAD branch. Scale bar: $1 \mathrm{~mm}$. Row I is a reprint from [24], with permission. 
images because of the intrinsically limited resolution and low soft tissue contrast. On the contrary, plaque types can be classified based on optical scattering contrast of different tissue types: 1) signal-rich regions from 3 o' clock to 6 o'clock in Fig. $7 \mathrm{Ib}$ indicate a fibrous plaque; and 2) a homogenous-boundary, signalpool region from 7 o'clock to 10 o'clock in Fig. 7 IIIb represents a lipid pool [46]. Automatic and quantitative classification of plaque types has been proposed by using algorithms [47, 48]. On the other hand, with limited penetration depth, OCT image cannot provide a clear visualization of media and adventitia layers at this intima-thickening coronary segment. The corresponding histology images (Fig. 7 Ic, Fig. 7 IIc and Fig. 7 IIIc) are stained with hematoxylin and eosin (H\&E). Cells with abundant pale, vesicular cytoplasm and bland nuclei (Fig. 7 IIIc) are lipid-laden foamy macrophages which are also confirmed by positive stain in the CD 68 slide. The dense, compact eosinophilic fibers (Fig. 7 Ia) represent increased amounts of collagen seen in a fibrous plaque. These histology results confirm the classification of plaque types by the IVUS-OCT images.
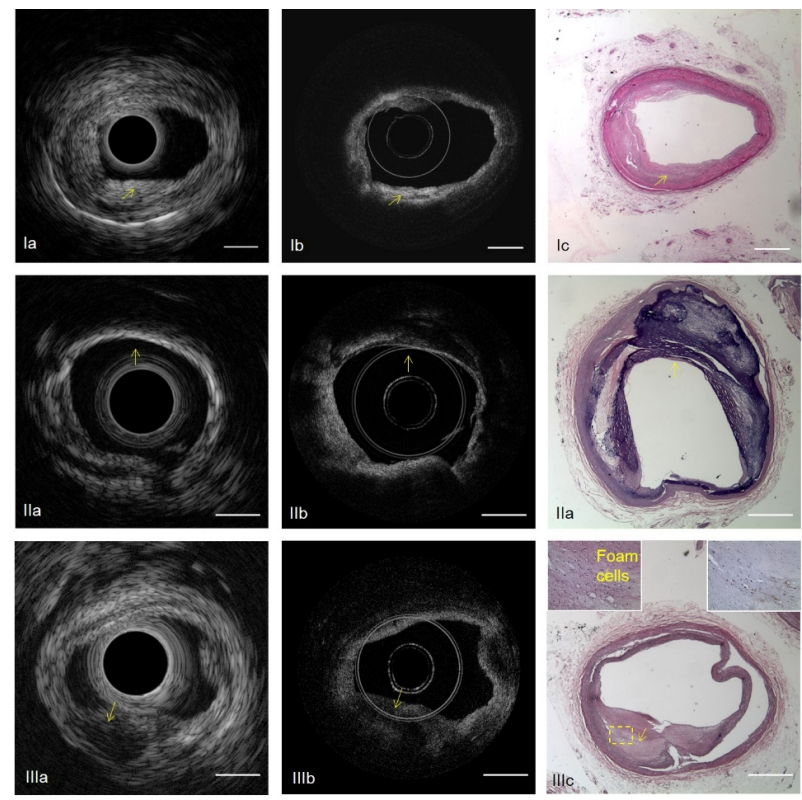

Fig. 7 Imaging of a coronary artery with a fibrous plaque (top row), a calcified plaque (middle row) and lipid plaque (bottom row). (Ia)(IIa)(IIIa) IVUS, (Ib)(IIb)(IIIb) OCT images, and (Ic)(IIc)(IIIc) corresponding histology image. Insets in (IIIc), high magnified images of the histology slides. Left inset, stained with H\&E; right inset, stained with CD68. Arrows denote the location of plaques. Scale bar: $1 \mathrm{~mm}$. Reprinted from [24], with permission.

Similarly, the ability to differentiate necrotic, calcified and fibrotic tissues was also evaluated in other studies [13, 31]. Quantitatively, the diagnostic accuracy comparison of IVUS-OCT diagnosis, with IVUS-only diagnosis and OCT-only diagnosis was conducted by using 241 plaque regions [33]. It was concluded that the use of IVUS and OCT is superior to IVUS-only and
OCT-only diagnosis for detection and characterization of coronary atherosclerotic plaques with high degree of sensitivity and specificity. In summary, IVUS-OCT shows promise to provide more accurate detection of different plaque types.

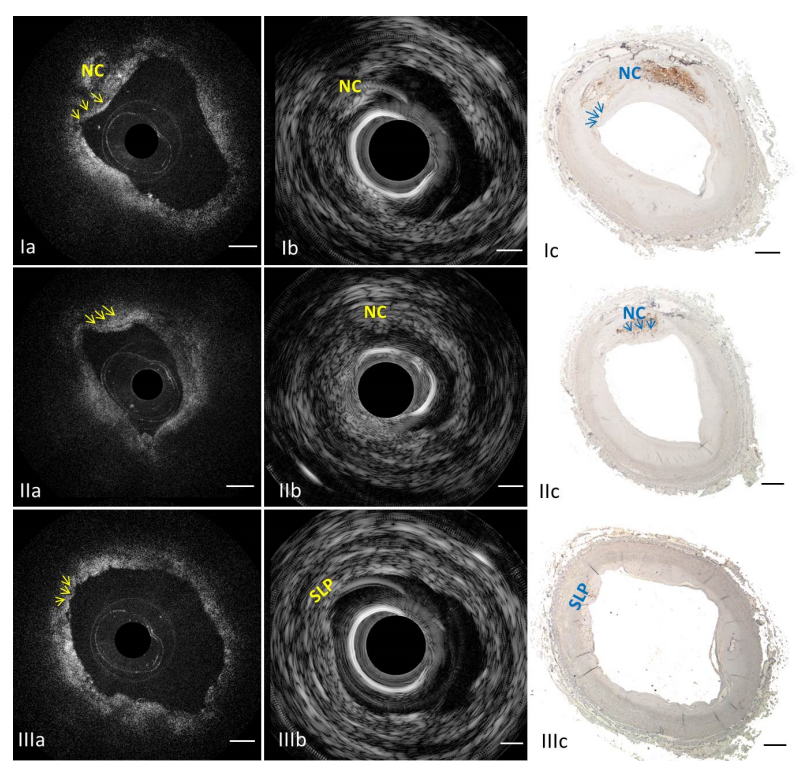

Fig. 8 Identification of vulnerable plaques by integrated IVUS-OCT system. First row: Example of a vulnerable plaque. (Ia) OCT image in which arrows point at the fibrous cap; (Ib) corresponding IVUS image indicates the location of necrotic core; (Ic) photo of the corresponding histology slide with CD 68 stain, highlighting macrophages and necrotic core. Middle row: A false positive case of vulnerable plaque diagnosis based on IVUS-only (IIb) was produced due to the insufficient resolution and sensitivity. Size of the thick cap can be determined by the corresponding OCT (IIa) and CD 68 histology (IIc). Bottom row: A false positive case of vulnerable plaque diagnosis based on OCT-only (IIIa) was produced due to OCT's limited penetration depth. A small lipid pool can be determined by IVUS (IIIb) and CD 68 histology (IIIc). Arrows denote the fibrous cap. NC: necrotic core; SLP: small lipid pool. Scale bar: $0.5 \mathrm{~mm}$. Reprinted from [26], with permission.

Accurate diagnosis of different plaque types is the first and foremost step for discerning a vulnerable plaque in which there is a core filled with necrotic tissues and a cap filled with fibrotic tissues.

\subsection{Identification of vulnerable plaques}

IVUS-OCT has the potential to address the need to identify vulnerable plaque in patients. Examples of vulnerable plaque and false vulnerable plaques, obtained by using an integrated IVUS-OCT system, are shown in Fig. 8. A vulnerable plaque can be easily diagnosed, as shown in the first row of Fig. 8. Arrows in the OCT image (Fig. 8 Ia) denote the thin fibrous cap while the size of the necrotic core can be estimated using the IVUS image (Fig. $8 \mathrm{Ib}$ ). In Fig. $8 \mathrm{IIb}$, 
physicians diagnosed this ROI as vulnerable plaques when only reading the IVUS image. This misdiagnosis was caused by the insufficient resolution and sensitivity of IVUS. However, using the OCT image as the reference (see Fig. 8 IIa), a signal low region with diffuse boundary (i.e., lipid plaque) could be visualized and the thickness of signal high region (fibrous cap) could be measured to be greater than $65 \mu \mathrm{m}$. In Fig. 8 IIIa, physicians diagnosed this ROI as vulnerable plaques when only reading the OCT image. This misdiagnosis was caused by OCT's limited penetration depth. However, using the IVUS image as the reference (see Fig. 8 IIIb), the small size of the lipid pool could be visualized.

\subsection{Evaluation of stent implantation}

IVUS-OCT images of a stent in a coronary artery is shown in Fig. 9. The 3D reconstruction of the entire pullback is shown in Figs. 9 IIa and IIb. There are two points in the clinical workflow where IVUS-OCT may play a key role in improving clinical outcomes: before and after stenting. IVUS-OCT may bring new insights into optimizing the selection and placement of stents and minimizing side effects.
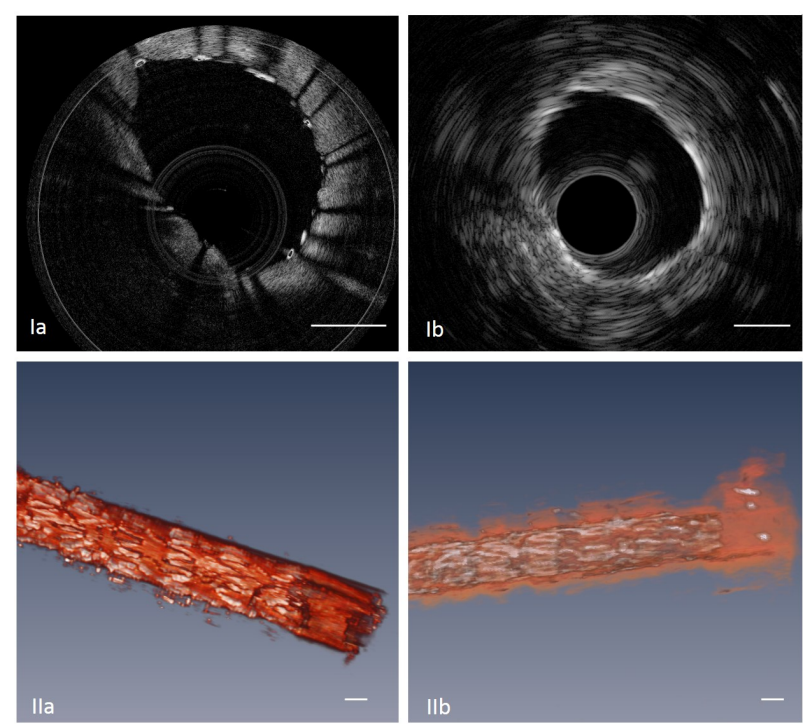

Fig. 9 IVUS-OCT imaging of a coronary artery with a stent implanted. OCT (Ia) IVUS (Ib) images, and OCT (IIa) IVUS (IIb) 3D rendering of a human coronary artery specimen with a stent. In (IIa) and (IIb), the white color coded places are stent struts and the red coded places are coronary artery tissue. Scale bar: $1 \mathrm{~mm}$.

In the previous section, the ability to accurately differentiate plaque types was discussed. Information, such as plaque compositions and lumen dimensions that are evaluated by IVUS-OCT, can assist cardiologists in optimizing the treatment plan. For instance, 1) heavily calcified lesions usually require more pressure to generate an adequate stent expansion than a softer lesion; 2) a lipid rich region is not a good landing point for a stent; 3) knowing the length of the lesion and the size of the lumen can help the physician select the optimal stent to use.

IVUS-OCT also provides important information after stenting, such as (1) evidence of inadequate stent expansion or strut apposition; (2) evidence of associated extensive vessel damage; and (3) evidence of the characteristics of neointimal hyperplasia in previously implanted stents [49]. Notably, there is a study that concluded that the best indicator to predict stent thrombosis, a deadly risk of stenting, was the ratio of uncovered to total stent struts [50]. By using IVUSOCT, visualization of stent struts and the surrounding tissues, with OCT's high resolution (to recognize uncovered struts and neoatherosclerosis that are not detectable by IVUS) [51] and IVUS's deep penetration depth (to image stents out of OCT image range or depth), can be achieved. IVUS and OCT provide complementary diagnostic values and may potentially contribute as a clinical benefit for patients $[12,52]$.

\subsection{Other clinical applications}

IVUS-OCT can also provide clinically valuable, complementary information for some other applications. For example, spontaneous coronary artery dissection is a disease that induces acute heart attack, but cannot be predicted and then prevented by using typically heart disease risk factors. Intravascular imaging is needed to make definitive diagnosis for this disease [53-55]. OCT was reported to allow the recognition of intimal tear while IVUS enables analysis of structures which are beyond the image range or depth of OCT, such as side branches, 3-layered membranes and dorsal structures [56].

\section{Future directions}

Since the first development and demonstration of integrated IVUS-OCT technology in 2010 [21, 30], many technical breakthroughs have been achieved in the past 5 years. However, more research and validation are required before this technology can be implemented into the daily clinical routine. The first and foremost step to achieve this goal is evaluating the diagnostic accuracy of IVUS-OCT in patient, which, however, is extremely costly. Due to the invasive nature of the technology and the vulnerability of coronary arteries (at where the imaging is performed), the in vivo human evaluation of this technology is classified as a significant risk and thus requires FDA approval for human study to be conducted in any U.S. research institute. The FDA approval requires significant resources for research institutes to perform such a clinical study. However, the involvement of medical device companies holds promise in overcoming this challenge. With an increased number of countries beginning to reimburse intravascular catheters and the fast growth of the Asia-Pacific market, the global market for IVUS itself has reach \$483.7 million in 2012 and is expected to reach $\$ 702$ million in 2017 (for a compound annual growth rate of $7.7 \%$ ) [57]. This fast 
growth encourages and enables more and more medical device companies to invest in new intravascular technologies, such as IVUS-OCT.

Another future direction of IVUS-OCT technology is to derive other imaging modalities from it and/or to combine with other imaging techniques so as to make it more powerful in identifying vulnerable plaques. The knowledge gained in developing IVUS-OCT technology can benefit a range of new imaging methods, such as intravascular photoacoustic (IVPA) and acoustic radiation force optical coherence elastography (ARFOCE). IVPA is expected to provide stronger chemical contrast to accurately identify lipid cores while ARFOCE holds potential to predict the rupture of a plaque by analyzing the stress generated by its necrotic pool.

Both IVUS and OCT signals are generated by detecting the scattering information of different plaque compositions. However, the scattering pattern produced by lipids may look similar to what is generated by another chemical composition. The diagnostic accuracy of IVUS in detecting lipid is limited [4]. Thus, a more accurate detection of the chemical composition of plaques may be of great interest to physicians. Near infrared spectroscopy (NIRS), which detects different molecules by their unique absorption properties, is currently under investigation in large-scale clinical studies [58, 59]. There is, however, a fundamental limitation. NIRS only provides 2D imaging of a coronary artery. To provide a 3D map of chemical compositions of a plaque, a trend of using PA for intravascular imaging has been seen in recent publications [60-64]. Although in vivo validation of IVPA in animals remains limited [61], we can expect a fast growth of IVPA in the near future by adopting the technical advancements of IVUS-OCT.

From a mechanical perspective, the rupture of a plaque happens when the stress on the plaque exceeds its tensile strength. The stress in the cap increases with decreasing thickness of the fibrous cap and increasing lipid-core burden. Thus, elastography, which assesses the mechanical properties, offers the opportunity to evaluate plaque vulnerability by analyzing its mechanical behavior. Several elastography technologies have been developed or are in development, such as ultrasound, MRI, laser speckle or OCT-based ones. The spatial resolutions of elastography techniques are determined by the underlying imaging modality. Accordingly, OCT-based elastography can provide much better resolution than MRI and US-based elastography [65-68]. To generate elastography, mechanical excitation of a tissue is required. The traditional excitation approach which compresses the subject statically/quasi-statically has limited imaging speed for in vivo imaging. Recently, ARFOCE was proposed [68-70], which use a dynamic excitation method. This novel technology enables a much higher imaging speed and makes it possible for in vivo imaging. When developing ARFOCE, we can borrow insights obtained in advancing IVUS-OCT technology since ARFOCE and IVUS-OCT both use ultrasound and OCT. It is promising that ARFOCE can be used intravascularly in the next couple of years to evaluate the mechanical property of a plaque.

\section{Conclusion}

The integrated IVUS-OCT technology is a powerful tool for diagnosing and characterizing vulnerable plaque. Since the first demonstration of IVUS-OCT in 2010, significant advances have been made. The fundamental challenge of IVUS-OCT technology has been overcome, and the speed of a fully-synchronized integrated system is now fast enough for safely imaging coronary arteries in vivo. Advancements have also been completed in catheter design: the imaging probe can be fabricated small enough for intracoronary imaging while the imaging quality is well maintained. In vivo animal studies validated the safety and effectiveness of this technology. Ex vivo studies using human coronary specimens demonstrated potential clinical applications of IVUS-OCT. Future directions of this technology include performing in vivo human studies and developing derivative imaging modalities.

\section{Acknowledgments}

We would like to thank many of our colleagues who have contributed to the intravascular imaging project the UCI Beckman Laser Institute, The Edwards Lifesciences Center for Advanced Cardiovascular Technology, Department of Biomedical Engineering, and School of Medicine, particularly Dr. P. M. Patel and his team at the UCI Division of Cardiology. In addition, we would like to thank our collaborators, Drs. Q. Zhou and K. K. Shung, as well as their students the USC NIH Ultrasonic Transducer Resource Center and Department of Biomedical Engineering, for miniature ultrasound transducers for the intravascular imaging project. Finally, this work was supported by the National Institutes of Health under grants R01-HL-125084, R01HL-127271, R01-HL-105215, R01-EY-021529, P41EB-002182, P41-EB-015890. Dr. Zhongping Chen has a financial interest in OCT Medical Inc., which, however, does not support this work. 